\title{
Chemical stratification in the Orion Bar: JCMT Spectral Legacy Survey observations
}

\author{
M. H. D. van der Wiel ${ }^{1,2}$, F. F. S. van der Tak ${ }^{2,1}$, V. Ossenkopf ${ }^{3,2}$, M. Spaans ${ }^{1}$, H. Roberts ${ }^{4}$, G. A. Fuller ${ }^{5}$, and R. Plume ${ }^{6}$ \\ 1 Kapteyn Astronomical Institute, PO Box 800, 9700 AV, Groningen, The Netherlands \\ e-mail: wiel@astro.rug.nl \\ 2 SRON Netherlands Institute for Space Research, PO Box 800, 9700 AV, Groningen, The Netherlands \\ I. Physikalisches Institut der Universität zu Köln, Zülpicher Straße 77, 50937 Köln, Germany \\ 4 Astrophysics Research Centre, School of Mathematics and Physics, Queen's University of Belfast, Belfast, BT7 1NN, UK \\ 5 Jodrell Bank Centre for Astrophysics, Alan Turing Building, University of Manchester, Manchester, M13 9PL, UK \\ 6 Department of Physics and Astronomy, University of Calgary, Calgary, T2N 1N4, AB, Canada
}

Received 21 November 2008 / Accepted 6 February 2009

\section{ABSTRACT}

\begin{abstract}
Context. Photon-dominated regions (PDRs) are expected to show a layered structure in molecular abundances and emerging line emission, which is sensitive to the physical structure of the region as well as the UV radiation illuminating it.

Aims. We aim to study this layering in the Orion Bar, a prototypical nearby PDR with a favorable edge-on geometry.

Methods. We present new maps of $2^{\prime} \times 2^{\prime}$ fields at $14^{\prime \prime}-23^{\prime \prime}$ resolution toward the Orion Bar in the SO $8_{8}-9_{9}, \mathrm{H}_{2} \mathrm{CO}_{5} 5_{15}-4_{14}$, ${ }^{13} \mathrm{CO} 3-2, \mathrm{C}_{2} \mathrm{H} 4_{9 / 2}-3_{7 / 2}$ and $4_{7 / 2}-3_{5 / 2}, \mathrm{C}^{18} \mathrm{O} 2-1$ and $\mathrm{HCN} 3-2$ transitions.

Results. The data reveal a clear chemical stratification pattern. The $\mathrm{C}_{2} \mathrm{H}$ emission peaks close to the ionization front, followed by $\mathrm{H}_{2} \mathrm{CO}$ and $\mathrm{SO}$, while $\mathrm{C}^{18} \mathrm{O}, \mathrm{HCN}$ and ${ }^{13} \mathrm{CO}$ peak deeper into the cloud. A simple PDR model reproduces the observed stratification, although the $\mathrm{SO}$ emission is predicted to peak much deeper into the cloud than observed while the $\mathrm{H}_{2} \mathrm{CO}$ peak is predicted to peak closer to the ionization front than observed. In addition, the predicted $\mathrm{SO}$ abundance is higher than observed while the $\mathrm{H}_{2} \mathrm{CO}$ abundance is lower than observed.

Conclusions. The discrepancies between the models and observations indicate that more sophisticated models, including production of $\mathrm{H}_{2} \mathrm{CO}$ through grain surface chemistry, are needed to quantitatively match the observations of this region.
\end{abstract}

Key words. ISM: molecules - ISM: structure - ISM: individual objects: Orion Bar - stars: formation

\section{Introduction}

In photon-dominated regions (PDRs), UV radiation between a few and $13.6 \mathrm{eV}$ drives the thermal and chemical balance of interstellar gas (Hollenbach \& Tielens 1999, and references therein). PDRs are ubiquitous in the universe: surfaces of molecular clouds adjacent to H II regions, planetary nebulae, protoplanetary disks and also the nuclei of distant active galaxies. As such, PDRs are signposts of radiative feedback processes driven by star formation. Heating proceeds through photo-electric emission from dust grains and cooling is mostly due to fine-structure emissions of $[\mathrm{CII}]$ and $[\mathrm{OI}]$, and $\mathrm{CO}$ rotational lines (e.g., Sternberg \& Dalgarno 1995; Kaufman et al. 1999; Meijerink \& Spaans 2005; Röllig et al. 2007). Shielding of the UV radiation by dust and gas creates a layering structure where a sequence of different chemical transitions is produced by the gradual attenuation of the UV field (Ossenkopf et al. 2007).

The Orion Bar is a prototypical PDR located between the Orion molecular cloud and the HII region surrounding the Trapezium stars, at a distance of $414 \mathrm{pc}$ (Menten et al. 2007). Multi-wavelength observations (Tielens et al. 1993; Van der Werf et al. 1996; Hogerheijde et al. 1995; Young Owl et al. 2000; Walmsley et al. 2000) indicate a geometry for the Bar where the PDR is wrapped around the H II region created by the Trapezium stars and changes from a face-on to an edge-on geometry where the molecular emissions peak. The mean density of the Bar is about $10^{5} \mathrm{~cm}^{-3}$, the mean molecular gas temperature $85 \mathrm{~K}$, and the impinging radiation field is $(1-4) \times 10^{4} \chi_{0}$
(Hogerheijde et al. 1995), where the Draine field $\chi_{0}=2.7 \times 10^{-3}$ erg s${ }^{-1} \mathrm{~cm}^{-2}$.

The clumpiness of the PDR inferred by Hogerheijde et al. (1995) was confirmed by interferometric data (Young Owl et al. 2000; Lis \& Schilke 2003). Clump densities up to $10^{7} \mathrm{~cm}^{-3}$ were derived by Lis \& Schilke (2003) while the density of the interclump medium should fall between a few $10^{4} \mathrm{~cm}^{-3}$ (Young Owl et al. 2000) and $2 \times 10^{5} \mathrm{~cm}^{-3}$ (Simon et al. 1997). The physical stratification of the PDR is well established (Tielens et al. 1993; Van der Werf et al. 1996; Simon et al. 1997; Lis \& Schilke 2003). Vibrationally excited $\mathrm{H}_{2}$ emission is located $15^{\prime \prime}$ from the ionization front, where $\mathrm{HCO}^{+} 1-0$ peaks as well, while the $\mathrm{CO}$ peak resides at $20^{\prime \prime}$ and CS slightly further in.

This paper presents emission maps of various molecular species, allowing us to probe and understand the chemical stratification in the Orion Bar in greater detail.

\section{Observations}

This work is based on data obtained as part of the Spectral Legacy Survey (SLS, Plume et al. 2007), being conducted at the James Clerk Maxwell Telescope ${ }^{1}$ on Mauna Kea, Hawai'i. The SLS is performing spectral imaging of $2^{\prime} \times 2^{\prime}$ fields in the

\footnotetext{
1 The James Clerk Maxwell Telescope is operated by The Joint Astronomy Centre on behalf of the Science and Technology Facilities Council of the United Kingdom, the Netherlands Organisation for
} Scientific Research, and the National Research Council of Canada. 
Table 1. Observed molecular lines.

\begin{tabular}{|c|c|c|c|c|c|c|c|c|c|c|c|}
\hline \multirow[t]{2}{*}{ Molecule } & \multirow{2}{*}{\multicolumn{3}{|c|}{ Transition }} & \multirow{2}{*}{$\begin{array}{l}\text { Frequency } \\
(\mathrm{MHz})\end{array}$} & \multirow{2}{*}{$\begin{array}{l}E_{\mathrm{up}} \\
(\mathrm{K})\end{array}$} & \multicolumn{3}{|c|}{ Peak position $(\mathrm{mpc})^{(d)}$} & \multirow{2}{*}{$\underset{\left(\mathrm{K} \mathrm{km} \mathrm{s}^{-1}\right)}{\int_{\mathrm{mb}} \mathrm{d} v^{(e)}}$} & \multicolumn{2}{|c|}{$N_{\mathrm{mb}}\left(\mathrm{cm}^{-2}\right)^{(g)}$} \\
\hline & & & & & & NE slice & SW slice & model & & $n=10^{5} \mathrm{~cm}^{-3}$ & LTE \\
\hline $\mathrm{C}_{2} \mathrm{H}$ & $N_{J}$ & $=4_{7 / 2}$ & $\rightarrow 3_{5 / 2}$ & $349400.5^{(a)}$ & 42 & $-14 \pm 1.8$ & $-23 \pm 1.0$ & -16 & $10.2 \pm 1.9$ & $5.5 \times 10^{14}$ & $2.7 \times 10^{14}$ \\
\hline $\mathrm{C}_{2} \mathrm{H}$ & $N_{J}$ & $=4_{9 / 2}$ & $\rightarrow 3_{7 / 2}$ & $349337.8^{(b)}$ & 42 & $-14 \pm 1.5$ & $-23 \pm 1.6$ & -16 & $13.5 \pm 2.4$ & $6.4 \times 10^{14}$ & $2.9 \times 10^{14}$ \\
\hline $\mathrm{O}-\mathrm{H}_{2} \mathrm{CO}$ & $J_{K p K o}$ & ${ }_{o}=5_{15}$ & $\rightarrow 4_{14}$ & 351768.6 & 62 & $-3 \pm 1.3$ & $-7 \pm 1.9$ & -12 & $11.1 \pm 1.9$ & $9.7 \times 10^{14}$ & $4.3 \times 10^{13}$ \\
\hline $\mathrm{SO}$ & $N_{J}$ & $=89$ & $\rightarrow 7_{8}$ & 346528.5 & 79 & $-2 \pm 1.4$ & $-5 \pm 1.0$ & 8 & $6.1 \pm 1.1$ & $1.5 \times 10^{15}$ & $7.7 \times 10^{13}$ \\
\hline $\mathrm{C}^{18} \mathrm{O}$ & $J$ & $=2$ & $\rightarrow 1$ & 219560.4 & 16 & $0 \pm 1.2$ & $0 \pm 1.4$ & 0 & $95.2 \pm 16$ & $1.5 \times 10^{16}$ & $1.8 \times 10^{16}$ \\
\hline $\mathrm{HCN}$ & $J$ & $=3$ & $\rightarrow 2$ & $265886.2^{(c)}$ & 26 & $3 \pm 2.5$ & $-1 \pm 1.8$ & 8 & $420 \pm 78$ & $8.1 \times 10^{15}$ & $4.4 \times 10^{14}$ \\
\hline${ }^{13} \mathrm{CO}$ & $J$ & $=3$ & $\rightarrow 2$ & 330588.0 & 32 & $4 \pm 1.9$ & $-1 \pm 3.0$ & 2 & $132 \pm 22$ & $>2.1 \times 10^{16(f)}$ & $>2.5 \times 10^{16(f)}$ \\
\hline
\end{tabular}

${ }^{(a)}$ Blend of $F=4-3$ and $F=3-2$ hyperfine components (separation $1.4 \mathrm{MHz}$ ). ${ }^{(b)}$ Blend of $F=5-4$ and $F=4-3$ hyperfine components (separation $1.3 \mathrm{MHz}$ ). ${ }^{(c)}$ Blend of $F=3-3, F=3-2$ and $F=2-1$ hyperfine components (total separation $1.5 \mathrm{MHz}$ ). ${ }^{(d)}$ Fitted position of the emission peak along the slice through the northeast and southwest cores (see Fig. 1), and the modeled slice (Fig. 3, bottom panel), all relative to the $\mathrm{C}^{18} \mathrm{O}$ peak. ${ }^{(e)}$ Fitted value of integrated intensity at peak position along the northeast slice (see Fig. 1); uncertainties include fitting uncertainty and $15 \%$ absolute calibration uncertainty. ${ }^{(f)}$ Column density uncertain due to high optical depth; values listed here are lower limits. ${ }^{(g)}$ Beam-averaged column densities for $n=10^{5} \mathrm{~cm}^{-3}$ and in LTE, both at $T=85 \mathrm{~K}$.

direction of the Orion Bar and four other targets. Once completed, the spectral range covered by the SLS will be 330$362 \mathrm{GHz}$. This Letter presents the first results from a selection of molecular lines in the already acquired data: two $\mathrm{C}_{2} \mathrm{H}$ lines and one transition each of $\mathrm{SO}, \mathrm{H}_{2} \mathrm{CO}$ and ${ }^{13} \mathrm{CO}$ between 330 and $350 \mathrm{GHz}$, and two lines in auxiliary data in the $230 \mathrm{GHz}$ window: $\mathrm{C}^{18} \mathrm{O} 2-1$ and HCN 3-2 (Table 1).

The SLS uses the 16-pixel HARP receiver $(325-375 \mathrm{GHz})$ and the ACSIS correlator. The angular resolution of the JCMT at the observed frequencies is $14-15^{\prime \prime}(\approx 30 \mathrm{mpc}$ at the distance of the Orion Bar); the harp4_mc jiggle position switch mode

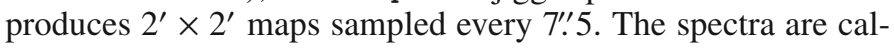
ibrated by observations at an off-position $10^{\prime}$ to the southeast. At an exposure time of 4 min per map pixel, the rms noise level in the current SLS data is $0.07-0.08 \mathrm{~K}$ in velocity bins of $0.83-$ $0.89 \mathrm{~km} \mathrm{~s}^{-1}$. Single sideband system temperatures for these observations are $\sim 700 \mathrm{~K}$.

The HCN 3-2 and $\mathrm{C}^{18} \mathrm{O} 2-1$ lines were observed in 2005 with the $A$-band $(215-275 \mathrm{GHz})$ receiver and the DAS spectrometer. The same $2^{\prime} \times 2^{\prime}$ field as with HARP was mapped at half-beamwidth spacing, using the raster mapping mode. The JCMT beam size at $220-266 \mathrm{GHz}$ is $19-23^{\prime \prime}(\approx 40 \mathrm{mpc})$. The offposition for the $A$-band observations was at $20^{\prime}$ southeast of the map center. The $\mathrm{HCN}$ and $\mathrm{C}^{18} \mathrm{O}$ observations reach a noise level of $0.5-0.6 \mathrm{~K}$ at a spectral resolution of 0.18 and $0.21 \mathrm{~km} \mathrm{~s}^{-1}$, respectively. System temperature values are $\sim 350 \mathrm{~K}$ (DSB); the integration time is $19 \mathrm{~s}$ per map pixel. The pointing accuracy was checked every hour and was generally better than $2^{\prime \prime}$ during all observing runs.

The data were manually reduced using standard Starlink procedures. The SLS observations were handled in blocks of $1.6 \mathrm{GHz}$ effective bandwidth and the other data as one set per emission line. The time series were converted to data cubes and first-order polynomial baselines were subtracted. Line intensities quoted here are given in terms of the main-beam temperature $T_{\mathrm{mb}}$, i.e., they have been corrected for the JCMT main-beam efficiency of $63 \%$ in the $345 \mathrm{GHz}$ window and $69 \%$ in the $230 \mathrm{GHz}$ window. Single-channel signal-to-noise ratios at the peak in the northeast slice are $\sim 16$ for $\mathrm{C}^{18} \mathrm{O}$ and $\mathrm{SO}, \sim 40$ for $\mathrm{HCN}, \mathrm{H}_{2} \mathrm{CO}$ and $\mathrm{C}_{2} \mathrm{H}$, and 900 for ${ }^{13} \mathrm{CO}$.

\section{Results}

Figure 1 presents images of the observed molecular emission, overlaid on an $8 \mu \mathrm{m}$ image of the Orion Bar taken with the
Spitzer Space Telescope (Megeath et al., in prep.). The midinfrared emission traces warm dust at the surface layer between the $\mathrm{H}$ II region and the molecular cloud. The molecular emission is seen to peak to the southeast of this layer, and the JCMT data reveal a clear stratification. The $\mathrm{C}_{2} \mathrm{H}$ emission peaks close to the surface layer, followed by $\mathrm{SO}$ and $\mathrm{H}_{2} \mathrm{CO}$, whereas $\mathrm{CO}$ and $\mathrm{HCN}$ peak deep into the molecular cloud.

The stratification is more easily visible in Fig. 2 which shows slices through the images in Fig. 1, taken along the arrows in Fig. 1, perpendicular to the ionization front. To produce these intensity profiles, all maps are regridded to 3 '.75 pixels, roughly half the pixel size of the original maps. Fitted peak positions and peak fluxes for the seven profiles are listed in Table 1, along with the spectroscopic parameters of the observed lines; the position uncertainties include Gaussian fitting errors $\left(<1^{\prime \prime} \approx 2 \mathrm{mpc}\right)$.

In addition to the stratification, the molecular emission shows a two-peak structure. The northeast peak is brighter in $\mathrm{HCN}$ and $\mathrm{H}_{2} \mathrm{CO}$, while the southwest peak is brighter in $\mathrm{SO}$ and the peak brightness is roughly equal for $\mathrm{CO}$ and $\mathrm{C}_{2} \mathrm{H}$. These variations are probably caused by variations in the filling factor of dense gas, as seen, e.g., in the high-resolution data by Lis \& Schilke (2003). The measured line widths of $5-8 \mathrm{~km} \mathrm{~s}^{-1}$ will be useful for future detailed modeling, however, this paper focuses on integrated intensities.

We use the observed line intensities and the radiative transfer program RADEX (Van der Tak et al. 2007) to estimate beamaveraged molecular column densities. Although temperatures are known to range from $50 \mathrm{~K}$ in dense clumps up to $150 \mathrm{~K}$ in the interclump medium (Batrla \& Wilson 2003; Lis \& Schilke 2003), we assume a kinetic temperature of $85 \mathrm{~K}$ and a volume density of $10^{5} \mathrm{~cm}^{-3}$, as applicable to the extended molecular gas (Hogerheijde et al. 1995). Since some of the line emission may originate from dense clumps, we also list column densities estimated in the high-density (LTE) limit (Table 1). Both for the $10^{5} \mathrm{~cm}^{-3}$ case as well as the LTE limit, varying the temperature by $\pm 30 \mathrm{~K}$ changes the column densities by $\lesssim 30 \%$ (where a higher temperature gives a lower column density), so the molecular column densities are reasonably well constrained.

Our values for the column densities agree with previously published values to a factor of $\sim 2$ : Hogerheijde et al. (1995) for $\mathrm{H}_{2} \mathrm{CO}, \mathrm{C}^{18} \mathrm{O}$ and $\mathrm{HCN}$, Jansen et al. (1995) for $\mathrm{C}_{2} \mathrm{H}$, and Leurini et al. (2006) for SO. The published column densities for $\mathrm{HCN}$ and SO correspond to our high-density (LTE) estimates (Table 1); this is consistent with these two species being confined to the high-density gas while the $\mathrm{H}_{2} \mathrm{CO}$ emission has 


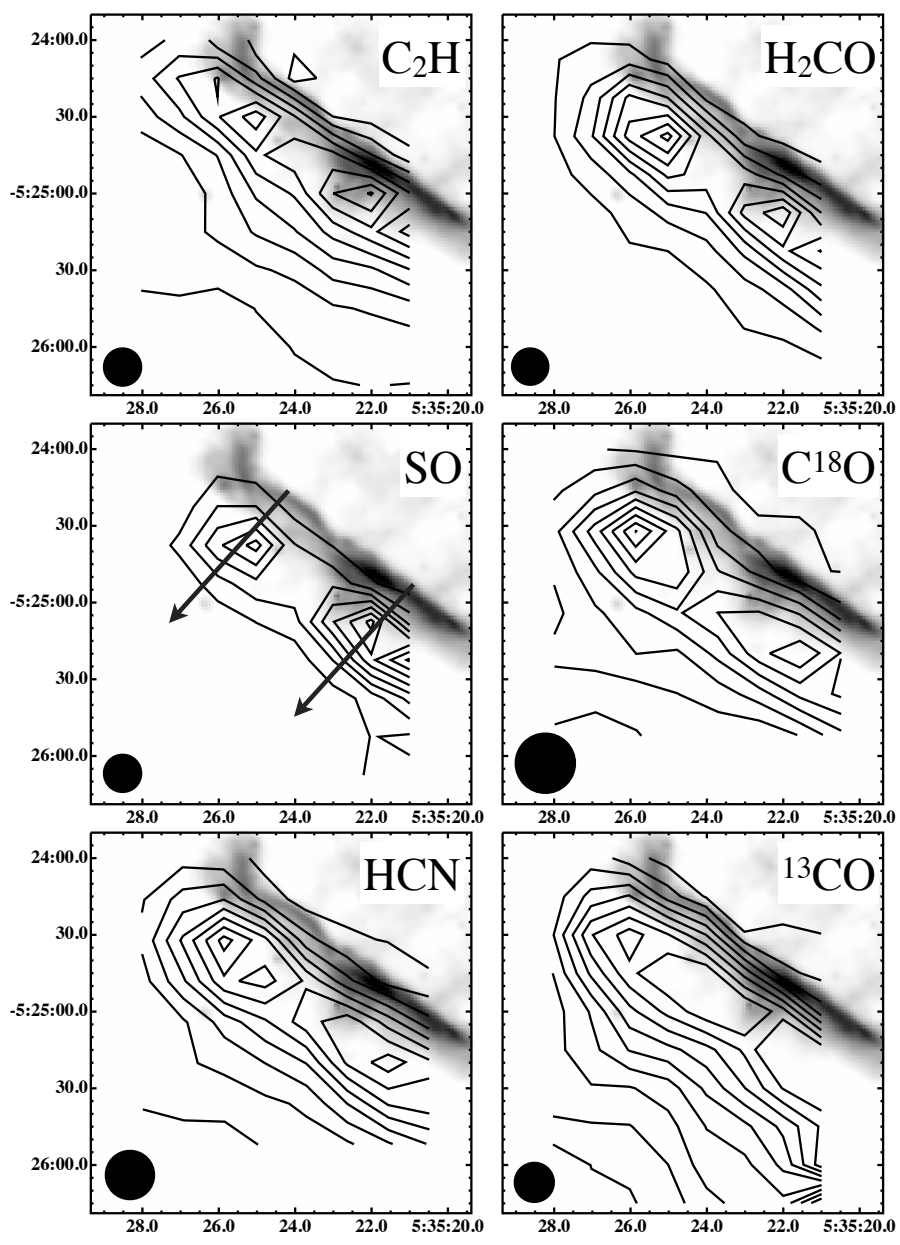

Fig. 1. Images of molecular emission, observed with the JCMT toward the Orion Bar of the species and transitions listed in Table 1. Line intensities are integrated over $5-8 \mathrm{~km} \mathrm{~s}^{-1}$, depending on the line width. The $\mathrm{C}_{2} \mathrm{H}$ transition shown here is the $4_{9 / 2}-3_{7 / 2}$ transition; the other transition shows a similar spatial distribution. Contour levels are drawn at $10,20, \ldots, 90 \%$ of the maximum integrated intensity for every map: $15.9 \mathrm{~K} \mathrm{~km} \mathrm{~s}^{-1}$ for $\mathrm{C}_{2} \mathrm{H}, 10.4$ for $\mathrm{H}_{2} \mathrm{CO}, 9.1$ for $\mathrm{SO}, 93.5$ for $\mathrm{C}^{18} \mathrm{O}$, 400.0 for $\mathrm{HCN}$, and 196.6 for ${ }^{13} \mathrm{CO}$. Note that the measured maximum $\int T_{\mathrm{mb}} \mathrm{d} v$ values across the northeast slices (Table 1) are not the same as the highest value across the entire map. The grayscale background image shows Spitzer Space Telescope $8 \mu \mathrm{m}$ continuum emission. The filled circle in each frame indicates the beam size at the relevant frequency. Axes are annotated with right ascension and declination (J2000).

contributions from both density phases. The spatial distribution of the emission in this work is similar to that of previously observed single-dish emission from molecules such as ${ }^{12} \mathrm{CO},{ }^{13} \mathrm{CO}$, $\mathrm{HCO}^{+}, \mathrm{H}_{2}, \mathrm{CN}$ and CS (e.g., Tauber et al. 1994; Van der Werf et al. 1996; Simon et al. 1997).

To convert the observed $\mathrm{C}^{18} \mathrm{O}$ line intensity to a total $\mathrm{H}_{2}$ column density, we assume an isotopic ratio ${ }^{16} \mathrm{O} /{ }^{18} \mathrm{O}=500$ (Wilson \& Rood 1994) and a CO abundance of $1.1 \times 10^{-4}$ as applicable for the Orion Bar PDR (Johnstone et al. 2003). The result is $N\left(\mathrm{H}_{2}\right)=6.8 \times 10^{22} \mathrm{~cm}^{-2}$, which implies the following molecular abundances at the peak locations: $x\left(\mathrm{C}_{2} \mathrm{H}\right)=4 \times 10^{-9}$; $x\left(\mathrm{H}_{2} \mathrm{CO}\right)=6 \times 10^{-10} ; x(\mathrm{SO})=1 \times 10^{-9} ; x(\mathrm{HCN})=6 \times 10^{-9}$. Here we have assumed that the emission from all molecules except $\mathrm{CO}$ originates in the high-density gas. Based on Jansen et al. (1995), who propose the clumps to encompass $10 \%$ of the material along every line of sight, we estimate the clumps to provide for roughly half of the total column density for those
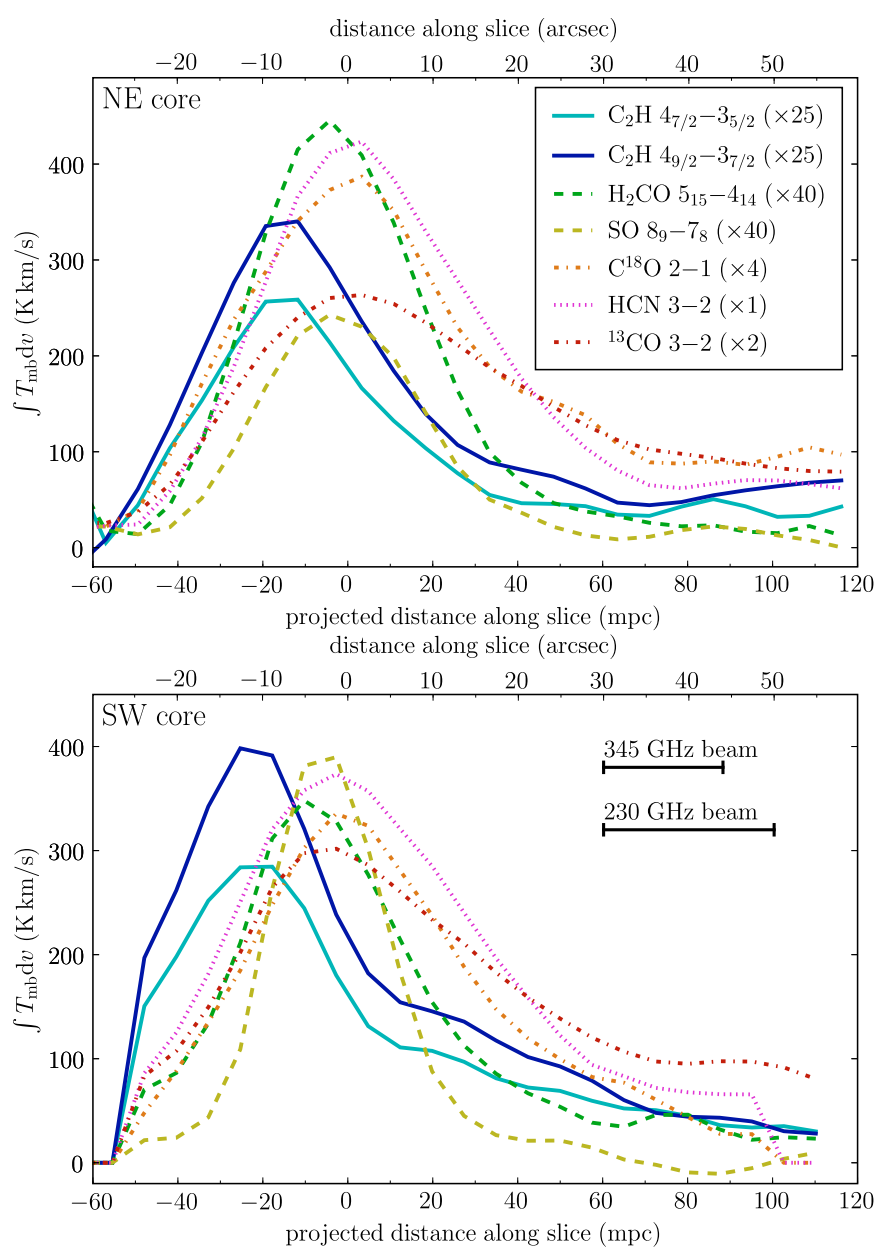

Fig. 2. Profiles of molecular emission along slices perpendicular to the ionization front at position angle $225^{\circ}$ (see arrows in Fig. 1): (top panel) through the northeast core; (bottom panel) through the southwest core. The zero point of the distance scale is placed at the $\mathrm{C}^{18} \mathrm{O}$ peak position: $\left(\mathrm{RA}=5^{\mathrm{h}} 35^{\mathrm{m}} 25^{\mathrm{s}} \cdot 4, \delta=-5^{\circ} 24^{\prime} 37^{\prime \prime}\right)$ for the northeast slice and (RA = $\left.5^{\mathrm{h}} 35^{\mathrm{m}} 21^{\mathrm{s}} \cdot 6, \delta=-5^{\circ} 25^{\prime} 19^{\prime \prime}\right)$ for the southwest slice.

molecules that exist in both phases. Hence, the resulting uncertainty in the derived abundances would not exceed a factor 2 . Since $\mathrm{C}_{2} \mathrm{H}$ and $\mathrm{CO}$ are hardly coexistent, the $\mathrm{C}_{2} \mathrm{H}$ abundance is probably underestimated by a factor of 3-10.

\section{Discussion}

To interpret our observations we compare them to the predictions from a PDR model. Previous models of the Orion Bar do not present abundances for all six species in our data, so we use the global conditions of the $\operatorname{Bar}\left(\chi=3 \times 10^{4} \chi_{0}, n_{\mathrm{H}}=10^{5} \mathrm{~cm}^{-3}\right.$, see Sect. 1) to construct a simple model using the KOSMA- $\tau$ PDR code (Röllig et al. 2006). We refer to Röllig et al. (2007) for a comparative study of other PDR codes. To simulate the plane-parallel geometry of the Orion Bar we represent it by one side of a very large sphere. Starting from atomic abundances of $x(\mathrm{O})=3.0 \times 10^{-4}, x(\mathrm{C})=1.4 \times 10^{-4}, x(\mathrm{~S})=2.8 \times 10^{-5}$ and $x(\mathrm{~N})=1.0 \times 10^{-4}$ (see also Cubick et al. 2008, Table 3), the model computes the equilibrium chemistry and temperature of the cloud using reaction rates from the UMIST database (Woodall et al. 2007). Based on the geometry in Fig. 1 of Jansen et al. (1995) we simulate the PDR as a layer of thickness $49 \mathrm{mpc}$ where the Bar represents a part of the layer that is parallel to the line of sight, so that its apparent depth is increased by a factor 

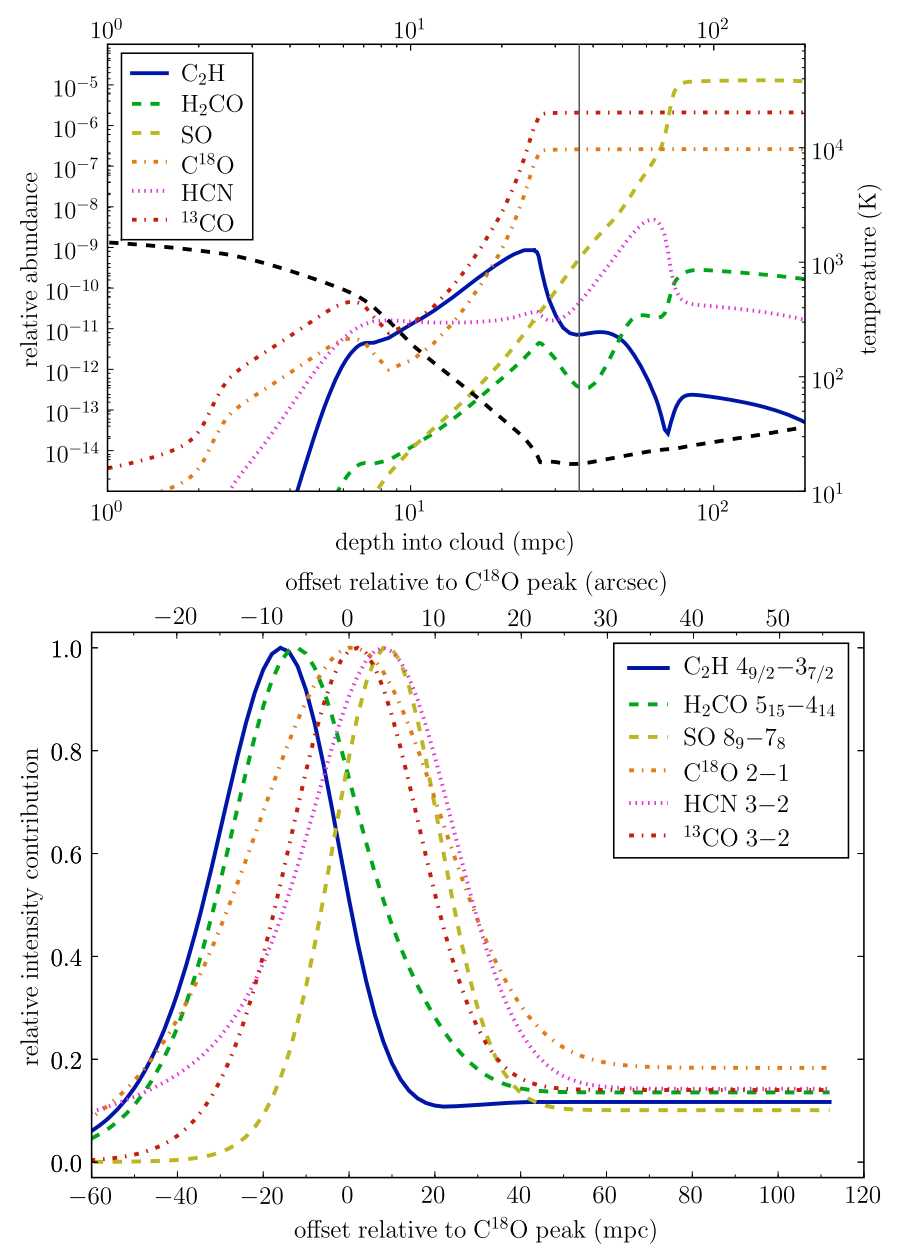

Fig. 3. Top panel: model calculation of relative abundances as a function of depth into the cloud. The dashed black curve shows the temperature profile. The thin vertical line indicates the zeropoint of the horizontal scale in the bottom panel. Bottom panel: modeled relative intensity profiles in the direction perpendicular to the line of sight, convolved by the JCMT beam. In this panel, the zeropoint of the depth scale is set to the modeled $\mathrm{C}^{18} \mathrm{O}$ peak, at $36 \mathrm{mpc}$ from the edge of the molecular cloud.

10 compared to its thickness. We note that the critical densities are $\sim 10^{7} \mathrm{~cm}^{-3}$ for all transitions in Table 1 except those of the $\mathrm{CO}$ isotopes, while the highest $\mathrm{H}_{2}$ density reported by Lis \& Schilke (2003) is also $10^{7} \mathrm{~cm}^{-3}$. Hence, to approximate the spatial distribution of the line emission, we assume optically thin LTE and weight the abundance profiles with a Boltzmann cutoff: $I_{\text {rel }}=\left(x / x_{0}\right) \exp \left(1-T_{\text {gas }} / E_{\text {up }}\right)$ if $T_{\text {gas }}<E_{\text {up }}$ and $I_{\text {rel }}=x / x_{0}$ otherwise. Here $I_{\text {rel }}$ is a normalized intensity, $x$ is abundance, $T_{\text {gas }}$ is the modeled local gas temperature (see Fig. 3, top panel), $E_{\text {up }}$ is given in Table 1 and the normalization factor $x_{0}$ is chosen from line to line. The distribution is convolved with the JCMT beam at the appropriate frequencies to obtain the predicted relative intensity profiles (Fig. 3, bottom panel).

Table 1 compares modeled positions of the peak emission to fitted peak positions in the observed profiles for each species. Overall our simple PDR model matches the observed layering of $\mathrm{C}_{2} \mathrm{H}-\mathrm{H}_{2} \mathrm{CO}-\mathrm{CO}$. For $\mathrm{H}_{2} \mathrm{CO}$ the highest abundance occurs in the cold shielded material, but the warm outer layers dominate the emission due to the higher $E_{\mathrm{up}}$ for the observed transition.

There are also significant discrepancies between the model and the observations. (i) The model predicts that SO peaks deep in the cold material (at $8 \mathrm{mpc}$ ), following the distribution of $\mathrm{HCN}$, not at the location where $\mathrm{H}_{2} \mathrm{CO}$ peaks (in front of $\mathrm{CO}$ ) as is observed. (ii) Conversely, the $\mathrm{H}_{2} \mathrm{CO}$ peak in both slices is observed closer to the peaks of the $\mathrm{CO}$ isotopes than to the $\mathrm{C}_{2} \mathrm{H}$ peak, while the model predicts $\mathrm{H}_{2} \mathrm{CO}$ to peak significantly closer to $\mathrm{C}_{2} \mathrm{H}$. (iii) For $\mathrm{HCN}$ the model peaks $5-9$ mpc deeper than is observed and (iv) the observations show an HCN emission wing toward the PDR surface which is weaker in the model. (v) We find that the absolute abundance of $\mathrm{HCN}$ at the model peak is in agreement with the observations, while the model abundances of $\mathrm{H}_{2} \mathrm{CO}$ and $\mathrm{C}_{2} \mathrm{H}$, respectively, are 2 and $\sim 4$ times smaller than observed. (vi) In addition, our modeled SO abundance is orders of magnitude larger than the observed value.

The underestimate of the $\mathrm{C}_{2} \mathrm{H}$ abundance predicted by our pure gas-phase model might be explained by $\mathrm{C}_{2} \mathrm{H}$ being formed via photo-destruction of PAH molecules, as proposed by Pety et al. (2005). This explains a detectable $\mathrm{C}_{2} \mathrm{H}$ abundance in the outer layers where the PAH emission peaks (Van der Werf et al. 1996).

Our modeled SO abundance is a factor $10^{4}$ larger than observed. This is explained by the too high gas phase sulfur abundance of $2.8 \times 10^{-5}$ used in the KOSMA- $\tau$ model, which represents diffuse clouds and no depletion (Federman et al. 1993). Recent estimates of cosmic sulfur abundance provide values as low as $1.4 \times 10^{-5}$ (Asplund et al. 2005) and sulfur depletion factors in dense clouds may range from 4 (Goicoechea et al. 2006) to 1000 (Tieftrunk et al. 1994). Depletion factors of several $10^{3}$ would be required to match the Orion Bar observations with the current model. The low depletion seems not to apply to the entire Orion region, but to be specific for the Horsehead, which has a particularly low density.

The large observed abundance of $\mathrm{H}_{2} \mathrm{CO}$ might be explained by the evaporation of molecules from warm $(\sim 30 \mathrm{~K})$ dust grains. Based on reaction rates from Woodall et al. (2007), the photodissociation lifetime for $\mathrm{H}_{2} \mathrm{CO}$ at $A_{\mathrm{V}}=5 \mathrm{mag}$ is only $\sim 10 \mathrm{yr}$, which introduces the need for a continuous supply of $\mathrm{H}_{2} \mathrm{CO}$, e.g., from grain surfaces (Herbst 2000). This is consistent with the suggestion by Leurini et al. (2006) that $\mathrm{H}_{2} \mathrm{CO}$ may originate primarily from the warm interclump medium.

Finally, the observed broad spatial profiles for $\mathrm{HCN}$ and ${ }^{13} \mathrm{CO}$ (Fig. 2) may be explained by optical depth effects, since the optical depth inferred by RADEX is $5-10$ for these molecules and $<1$ for the others. The different spatial distributions of the two CO isotopologs result mainly from the different beam sizes.

Future observations will test our hypothesis that grain surface chemistry plays a key role in PDRs. One such test will be JCMT-SLS observations of $\mathrm{CH}_{3} \mathrm{OH}$ in the Orion Bar: while Leurini et al. (2006) suggest that $\mathrm{CH}_{3} \mathrm{OH}$ traces the clumps and $\mathrm{H}_{2} \mathrm{CO}$ traces the interclump medium, both molecules are thought to be the products of $\mathrm{CO}$ hydrogenation on ice (Watanabe et al. 2004), so their spatial coincidence would mark a critical validation step. Other analysis will explore sulfur chemistry using sulfur-bearing species covered by the SLS, such as CS and $\mathrm{SO}_{2}$. Once completed, the SLS data set will enable studies of multiple transitions of various molecules, so that the excitation of molecules (as well as their velocity structure) can also be compared to models. In the longer term, (sub)millimeter-wave imaging spectroscopy of other Galactic PDRs is needed to establish how common grain chemistry is and how it depends on environment. Important tests will be spectral surveys with the HIFI instrument on board ESA's Herschel Space Observatory, which will target hundreds of molecular lines in many Galactic PDRs and allow a detailed picture of their chemistry to emerge.

Acknowledgements. The authors acknowledge the JCMT staff for their support, Markus Röllig for help with the PDR model, and Peter Schilke, Maryvonne Gerin, and an anonymous referee for their careful reading of the manuscript. 


\section{References}

Asplund, M., Grevesse, N., \& Sauval, A. J. 2005, in Cosmic Abundances as Records of Stellar Evolution and Nucleosynthesis, ed. T. G. Barnes, III, \& F. N. Bash, ASP-CS, 336, 25

Batrla, W., \& Wilson, T. L. 2003, A\&A, 408, 231

Cubick, M., Stutzki, J., Ossenkopf, V., Kramer, C., \& Röllig, M. 2008, A\&A, 488,623

Federman, S. R., Sheffer, Y., Lambert, D. L., \& Gilliland, R. L. 1993, ApJ, 413, L51

Goicoechea, J. R., Pety, J., Gerin, M., et al. 2006, A\&A, 456, 565

Herbst, E. 2000, in From Molecular Clouds to Planetary, ed. Y. C. Minh, \& E. F. van Dishoeck, IAU Symp., 197, 147

Hogerheijde, M. R., Jansen, D. J., \& Van Dishoeck, E. F. 1995, A\&A, 294, 792

Hollenbach, D. J., \& Tielens, A. G. G. M. 1999, Rev. Mod. Phys., 71, 173

Jansen, D. J., Spaans, M., Hogerheijde, M. R., \& Van Dishoeck, E. F. 1995, A\&A, 303, 541

Johnstone, D., Boonman, A. M. S., \& van Dishoeck, E. F. 2003, A\&A, 412, 157

Kaufman, M. J., Wolfire, M. G., Hollenbach, D. J., \& Luhman, M. L. 1999, ApJ, 527,795

Leurini, S., Rolffs, R., Thorwirth, S., et al. 2006, A\&A, 454, L47

Lis, D. C., \& Schilke, P. 2003, ApJ, 597, L145

Meijerink, R., \& Spaans, M. 2005, A\&A, 436, 397

Menten, K. M., Reid, M. J., Forbrich, J., \& Brunthaler, A. 2007, A\&A, 474, 515

Ossenkopf, V., Röllig, M., Cubick, M., \& Stutzki, J. 2007, in Molecules in Space and Laboratory, ed. J. L. Lemaire, \& F. Combes (S. Diana Publ.), 95
Pety, J., Teyssier, D., Fossé, D., et al. 2005, A\&A, 435, 885

Plume, R., Fuller, G. A., Helmich, F., et al. 2007, PASP, 119, 102

Röllig, M., Ossenkopf, V., Jeyakumar, S., Stutzki, J., \& Sternberg, A. 2006, A\&A, 451, 917

Röllig, M., Abel, N. P., Bell, T., et al. 2007, A\&A, 467, 187

Simon, R., Stutzki, J., Sternberg, A., \& Winnewisser, G. 1997, A\&A, 327, L9

Sternberg, A., \& Dalgarno, A. 1995, ApJS, 99, 565

Tauber, J. A., Tielens, A. G. G. M., Meixner, M., \& Goldsmith, P. F. 1994, ApJ, 422, 136

Tieftrunk, A., Pineau des Forets, G., Schilke, P., \& Walmsley, C. M. 1994, A\&A, 289,579

Tielens, A. G. G. M., Meixner, M. M., Van der Werf, P. P., et al. 1993, Science, 262, 86

Van der Tak, F. F. S., Black, J. H., Schöier, F. L., Jansen, D. J., \& Van Dishoeck, E. F. 2007, A\&A, 468, 627

Van der Werf, P. P., Stutzki, J., Sternberg, A., \& Krabbe, A. 1996, A\&A, 313, 633

Walmsley, C. M., Natta, A., Oliva, E., \& Testi, L. 2000, A\&A, 364, 301

Watanabe, N., Nagaoka, A., Shiraki, T., \& Kouchi, A. 2004, ApJ, 616, 638

Wilson, T. L., \& Rood, R. 1994, ARA\&A, 32, 191

Woodall, J., Agúndez, M., Markwick-Kemper, A. J., \& Millar, T. J. 2007, A\&A, 466, 1197

Young Owl, R. C., Meixner, M. M., Wolfire, M., Tielens, A. G. G. M., \& Tauber, J. 2000, ApJ, 540, 886 